\title{
Editorial
}

\section{The physiotherapy experience of managing patients with COVID-19}

\author{
Grace McKee ${ }^{\mathrm{a}}$, Catriona Cronin ${ }^{\mathrm{a}}$, Pedro Vasquez ${ }^{\mathrm{a}}$, Dearbhla Burke ${ }^{\mathrm{b}}$ and Deirdre O'Flaherty \\ ${ }^{a}$ Beaumont Hospital, Beaumont Road, Beaumont, Dublin, Ireland \\ ${ }^{\mathrm{b}}$ Clontarf Hospital, Clontarf, Dublin, Ireland
}

In January 2020, the WHO declared the SARSCoV-2 outbreak a public health emergency; by March 11th, a pandemic was declared [1]. To date in Ireland, over 4,118 patients have been admitted to acute hospitals as a result of infection with COVID-19 with over 524 of these requiring intensive care unit (ICU) support (numbers correct as of 22nd October 2020) (COVID Tracker). Beaumont Hospital, a large teaching hospital and tertiary care centre was one of the busiest hospitals in Ireland during March to June 2020 for COVID-19 admissions, during this period 174 patients were discharged from Beaumont $22 \%$ of whom had been admitted to the intensive care unit [2].

Physiotherapy was, and continues to be involved along the continuum of care for COVID-19 patients: from acute admission to ICU, and ward level management to rehabilitation at dedicated facilities. Treating

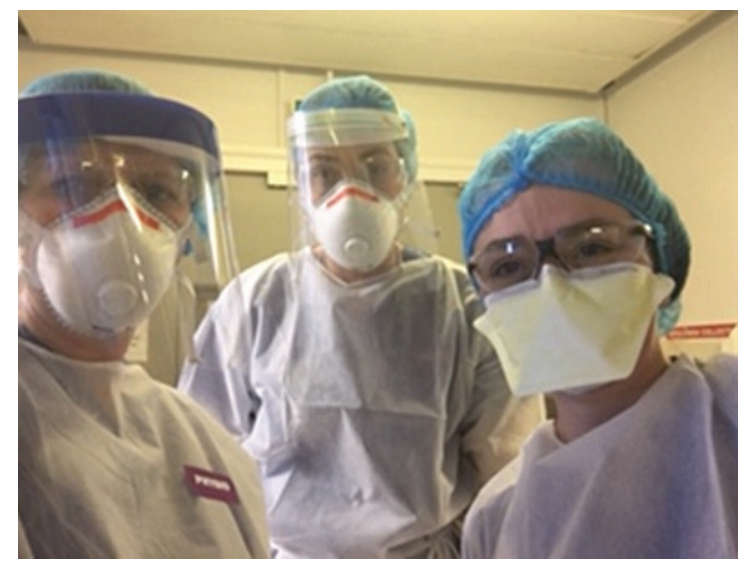

Fig. 1. Respiratory physiotherapists in PPE. a patient with COVID-19 posed many new challenges to physiotherapy, such as modification of treatment techniques to ensure safety for both the patient and staff. Preventing cross contamination exposure was paramount which involved physiotherapists working many hours in cumbersome personal protective equipment (PPE - Figs. 1, 2). The physiotherapy department at Beaumont Hospital was central to the management of these patients and had to quickly adapt to deal with the increased demand on service. The physiotherapy department moved from a five day eight hour service to a seven day service, providing 12 hour cover to the intensive care unit. Here we report the experience of the physiotherapy department at Beaumont Hospital and how they managed COVID-19 patients throughout their inpatient admission including modification of treatment techniques

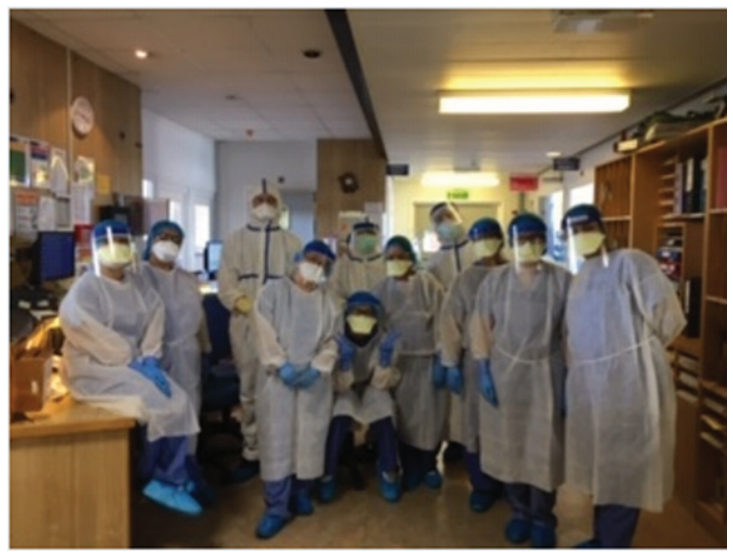

Fig. 2. Respiratory ward. 
and rehabilitation both on site and at a dedicated rehabilitation facility (Clontarf Hospital).

Cardiovascular (CV) instability and hypoxia are common in COVID-19 and these parameters are highly labile due to the ventilation perfusion (V/Q) disruption from the interstitial lung injury and $\mathrm{CV}$ effects of the COVID-19 virus [3, 4]. Proning is a technique frequently used to manage patients with Adult Respiratory Distress Syndrome (ARDS) to improve their V/Q matching (Faculty of Intensive Care Medicine) [5] and was shown to beneficial in managing patients with COVID-19 [6]. A 16 hour proning protocol was implemented in ICU and physiotherapists were trained to manage the proning regime (including self proning) in ICU and the wards.

From a respiratory physiotherapy perspective, patients with COVID-19 initially had low secretion load but patients may have become more productive in sub-acute stages of the disease. Physiotherapy adapted their traditional techniques to manage the cross contamination risk associated with secretion management to treat patients both on supportive devices and those self-ventilating. Respiratory physiotherapy practices for secretion management are deemed an aerosol generating procedure (AGP) [7]. This included tracheostomy care, non-invasive ventilation (NIV) and any physiotherapy adjunct that would stimulate a cough. Therefore these practices were modified accordingly. For example; patients who required NIV, had two specialist bacterial/viral filters, attached firstly to the NIV machines directly and the 2 nd to the patient interface to minimise aerosol exposure. All NIV masks were non-vented with an anti-asphyxiation exhalation port inserted below the 2nd filter, thus reducing the risk of small particle contamination to staff (Figs. 3, 4). Tracheostomy management included the use of closed circuit suctioning at ward level and prompt decannulation, bypassing the use of speaking valves or capping, common in weaning protocols. High Flow Nasal Oxygen (HFNO) was strictly managed by a Respiratory Consultant and kept at flows of 30L. All nebulised drugs were administered through Aerogen nebulisers. Full PPE was worn for all COVID-19 patients.

Rehabilitation of a COVID-19 patient started early in ICU, helping prevent critical illness myopathy/ neuropathy. Specialised ICU rehabilitation equipment such as a Combilyser and Letto Motomed bikes were used in conjunction with functional goal setting. Once extubated these patients were transferred to the respiratory ward for continued management of their

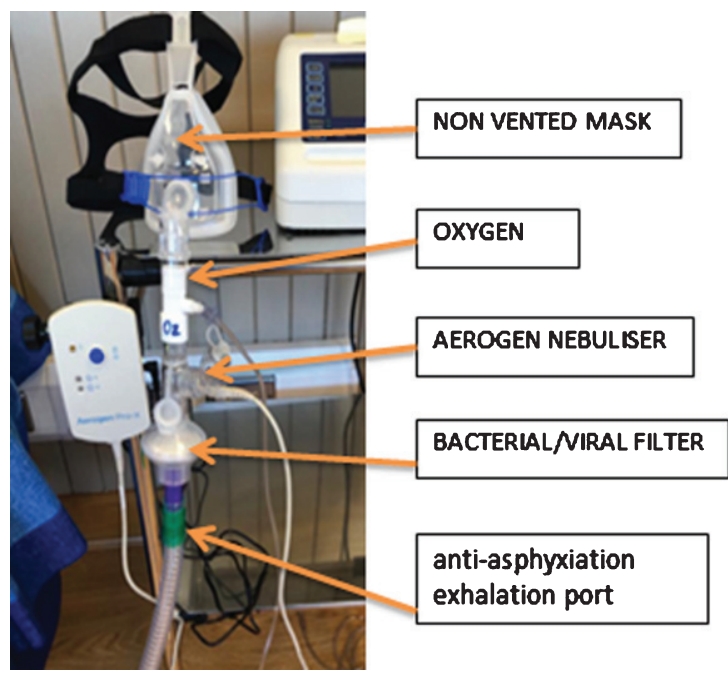

Fig. 3. NVI COVID circuit.

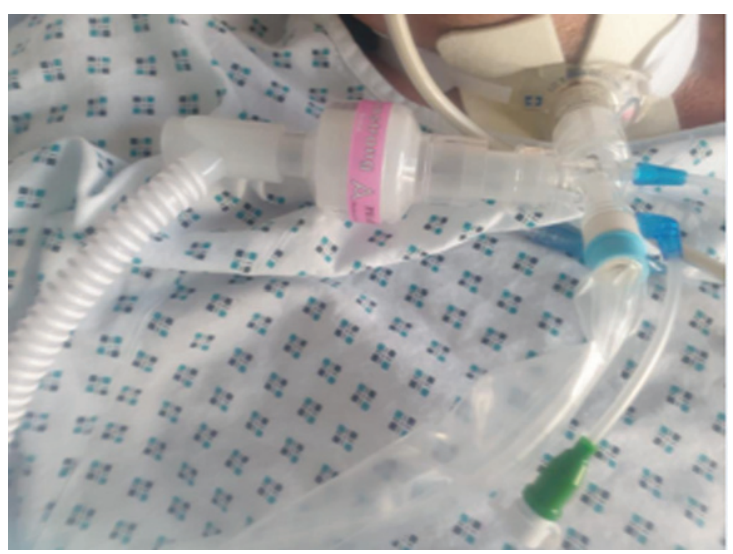

Fig. 4. Bacterial/Viral filter \& Closed Suction Circuit.

respiratory and rehabilitation needs. Physiotherapy managed patients on NIV, those with tracheostomies and those with ongoing high-level oxygen requirements. Some COVID-19 patients, whose ceiling of care excluded intubation were admitted directly to the respiratory ward and managed by physiotherapy with NIV, high oxygen support and proning. Patients with COVID-19 continued to experience episodes of significant desaturation and tachycardia with minimal activity [8]. Therefore all physiotherapy interventions both in ICU and on the ward were managed cautiously to ensure oxygen titration met the oxygen saturation demand throughout rehabilitation and CV parameters and oxygen saturation were closely monitored.

Once patients were deemed stable, but required further rehabilitation they were transferred to off-site 
facilities, including Clontarf Hospital. These patients often had complex needs, including myopathy, neuropathy, fatigue, anxiety, reduced exercise tolerance and ongoing hypoxia requiring supplemental oxygen therapy. Patients received an individually tailored rehabilitation program to meet their specific needs and goals. Specific measures were implemented to allow for safe rehabilitation of these patients whilst minimising the risk of transmission to staff and other patients: (i) reduced patient contact time (ii) all staff wore PPE (iii) patients had no to access to the physiotherapy gym (iv) no group rehabilitation classes, (v) social distancing in wards (reduced from 6 beds to 4 ), (vi) no visitors were allowed (vi) and shorter length of stay, similar to other countries [9], with limited opportunity for onward outpatient service referral as all were suspended in Ireland on the 16th March 2020. 'The Clontarf Hospital Discharge Support Service' was developed to provide telephone support for patients and their families, helping with the transition home. Patients were also provided with an advice and education booklet on discharge. A Post COVID Survivorship clinic has been established which aims to follow-up all COVID-19 patients who were admitted to Beaumont Hospital.

This editorial details the response of a physiotherapy department to COVID-19 during the "first wave." At the time of writing this piece we are in the "second wave" of this virus and anticipating the flu season. At the onset of the "first wave" of COVID19 , the physiotherapy community came together to share information which helped guide physiotherapy practice during the challenging time. We hope that through sharing our experience we can provide learning to others which may help the management of COVID-19 in the future. We also acknowledge the future learning that will take place as we continue to understand this virus and the long term health implications.

\section{References}

[1] World Health Organisation https://www.who.int/emergen cies/diseases/novel-coronavirus-2019/interactive-timeline [last accessed 29 $9^{\text {th }}$ October 2020]
[2] O'Brien H, Tracey MJ, Ottewill C, O'Brien ME, Morgan RK, Costello RW, Gunaratnam C, Ryan D, McElvaney NG, McConkey SJ, McNally C, Curley GF, MacHale S, Gillan D, Pender N, Barry H, de Barra E, Kiernan FM, Sulaiman I, Hurley K. An integrated multidisciplinary model of COVID19 recovery care. Irish Journal of Medical Science. 2020;1-8. Advance online publication. https://doi.org/10.1007/s11845020-02354-9

[3] Hendren NS, Drazner MH, Bozkurt B, Cooper LT, Jr. Description and Proposed Management of the Acute COVID-19 Cardiovascular Syndrome. Circulation. 2020; 141(23):1903-14. https://doi.org/10.1161/CIRCULATION AHA. 120.047349

[4] Frizzelli A, Tuttolomondo D, Aiello M, Majori M, Bertorelli $\mathrm{G}$, Chetta A. What happens to people's lungs when they get coronavirus disease 2019? Acta bio-medica : Atenei Parmensis. 2020;91(2):146-9. https://doi.org/10.23750/abm. v91i2.9574

[5] Faculty of Intensive Care Medicine and Intensive Care Society (2018). Guidelines on the management of acute respiratory distress syndrome. Version 1 July.

[6] Perier F, Tuffet S, Maraffi T, Alcala G, Victor M, Haudebourg AF, De Prost N, Amato M, Carteaux G, Mekontso Dessap A. Effect of PEEP and Proning on Ventilation and Perfusion in COVID-19 ARDS. American journal of respiratory and critical care medicine, (2020). 10.1164/rccm.202008-3058LE. Advance online publication. https://doi.org/10.1164/rccm. 202008-3058LE

[7] Health Service Executive (HSE) 2013 https://www.hse.ie/ eng/about/who/healthwellbeing/infectcont/sth/resources/aer osol-generating-procedures.pdf [last accessed 29th October 2020)

[8] Greenhalgh T, Javid B, Knight M, et al. What is the efficacy and safety of rapid exercise tests for exertional desaturation in COVID-19? Oxford COVID-19 Evidence Service, 2020. https://www.cebm.net/COVID-19/what-is-the-efficacy-andsafety-of-rapid-exercise-tests-for-exertional-desaturationin-COVID-19/

[9] Bettger JP, Thoumi A, Marquevich A, De Groote W, Battistella LR, Imamuara M, Delgado Ramos V, Wang N, Dreinhoefer KE, Manager A, Ghandi DBC, Ng YS, Lee KH, Ming JTW, Pua YH, Inzitari M, Mnbaga BT, Shayo MJ, Brown DA, Carvalho M, Oh-Park M, Stein J. COVID-19: maintaining essential rehabilitation services across the care continuum. BMJ Global Health. 2020;5:e002670. doi:10.1136/ bmjgh-2020-002670 